\title{
Interpolated elastic structure from the motion of dots
}

\author{
AKIRA ISHIGUCHI \\ University of Tokyo, Tokyo, Japan
}

\begin{abstract}
The ability of the human visual system to recover structure from the motion of dots was examined, using stimuli that produced the "rubber pencil illusion." These stimuli consisted of moving dots that simulated the motion of points on a wiggling rod. In Experiment 1, it was found that the dots were perceived to constitute an elastic or curved rod, although the motion of a rigid rod had been simulated. This elasticity was influenced, in particular, by the number of dots and by the phase difference between the motions of the dots. Experiment 2 was conducted to investigate the crucial factors that caused the perception of this elastic rod. The results showed that neither the distance between the dots nor the phase difference between the motions of the dots had a crucial role.
\end{abstract}

The human visual system can recover the spatial structure of an object from the motion of its elements. Most research on this capability has presupposed the rigidity principle, or the rigidity assumption in Ullman's (1979) terms. This assumption is based on the observation that the preferred interpretation of structure from motion is often the one in which the elements move together as a rigid object, rather than the one in which a collection of elements moves independently in space. The rigidity assumption solves the problem of the ambiguity that confronts the human visual system in the interpretation of structure from motion, and the structure of the object can be interpreted uniquely. Some results obtained to date have confirmed the feasibility of the rigidity assumption (Johansson, 1973, 1975; Ullman, 1979; Wallach \& O'Connell, 1953).

When the image's motion corresponds to that of a rigid 3-D object in motion, the human visual system must usually recover the rigid 3-D structure from unrestricted motions. The human visual system needs information to determine this structure, and a problem about the information required for the unique recovery of an object's structure must be raised. Ullman (1979) proved that four points in three orthographic views are sufficient for a unique interpretation of structure from motion. In additon, Tsai and Huang (1981) established that seven points in two perspective views guarantee a unique interpretation (see Ullman, 1983, for a review).

On the other hand, when certain limitations are imposed on the motion of the viewed objects-in particular, when the motion is restricted in a plane-the task turns out to

The author is indebted to T. Oyama of the University of Tokyo for his helpful suggestions about substance. The author also wishes to express his gratitude to K. Nakayama and S. Shimojo of the SmithKettlewell Institute of Visual Sciences, and J. M. Foley of the University of California, Santa Barbara, for their critical reading of and valuable comments on an earlier draft. The author's mailing address is Department of Psychology, Faculty of Letters, University of Tokyo, 7-3-1 Hongo, Bunkyo-ku, Tokyo 113, Japan. be somewhat easier, and the human visual system needs less information for the task. Hoffman and Flinchbaugh (1982) proved that for a rigid rod moving in a plane, its length and orientation in space were determined uniquely by three orthographic views of the two endpoints.

In the case of motion restricted to a plane, a widely known visual phenomenon can lead to an illusory interpretation of the structure from motion. This phenomenon is known as the "rubber pencil illusion," a term coined by Pomerantz (1983). It is "produced easily by wiggling a pencil that is held loosely and off-center between the thumb and index finger' (p. 365). Pomerantz concluded that the rubber pencil illusion was due to visual persistence, which probably occurred early in the human visual system, perhaps even in the retina. That is, the envelope that bounded the total trajectory for Pomerantz's display was curved at the top and bottom. This curvature produced by the visual persistence could, in turn, induce the perceived bending motion.

As mentioned above, Hoffman and Flinchbaugh (1982) proved mathematically the scheme of recovery of a rigid rod from the motion of its two endpoints. A pencil is a kind of rod. If we attach small lights to the endpoints of a pencil and wiggle it, what phenomenon do we observe? Do our visual systems recover the rigid pencil from the motion of its elements, or do we perceive a "rubber" pencil? The experiments reported here were conducted to investigate this question. The first experiment included two subexperiments in order to examine the effect of both the spatial variables and the velocity variable of the stimuli. The spatial variables used in this experiment were both the number of stimuli (dots) and the spatial scale.

\section{EXPERIMENT 1}

\section{Method}

\section{Subjects}

Fifteen undergraduates at the University of Tokyo participated as subjects in this experiment. All subjects had normal or correctedto-normal visual acuity. 


\section{Apparatus}

Stimulus patterns were generated by a microcomputer (NEC PC9801m) and displayed on a CRT (Kikusui COS1711 with a P31 phosphor). The CRT was placed $57.5 \mathrm{~cm}$ from the eyes of the subject, who sat in a chair with a chinrest in a dimly lighted room.

\section{Stimuli}

Experiment 1A: Spatial Factor. A set of 25 displays was presented in random order. Each display was presented three times. A sample display is illustrated in Figure 1. The broken lines indicate the simulated wiggling rigid pencil. They were not present in the actual displays. The $x-y$ coordinates of each dot in motion as a function of time $(t)$ can be described by the following equations [where $\mathrm{D} 1 x(t)$ is the $x$ coordinate of $\mathrm{D} 1$, etc.]

$$
\begin{aligned}
& \mathrm{D} 1 x(t)=\text { constant } \\
& \mathrm{D} 1 y(t)=a \sin (w t+s 1) \\
& \mathrm{D} 2 x(t)=\mathrm{D} 1 x(t)+d \cos [s 2 \sin (w t)] \\
& \mathrm{D} 2 y(t)=\mathrm{D} 1 y(t)+d \sin [s 2 \sin (w t)] \\
& \mathrm{D} 3 x(t)=[\mathrm{D} 1 x(t)+\mathrm{D} 2 x(t)] / 2 \\
& \mathrm{D} 3 y(t)=[\mathrm{D} 1 y(t)+\mathrm{D} 2 y(t)] / 2 \\
& \mathrm{D} 4 x(t)=[2 \mathrm{D} 1 x(t)+\mathrm{D} 2 x(t)] / 3 \\
& \mathrm{D} 4 y(t)=[2 \mathrm{D} 1 y(t)+\mathrm{D} 2 y(t)] / 3 \\
& \mathrm{D} 5 x(t)=[\mathrm{D} 1 x(t)+2 \mathrm{D} 2 x(t)] / 3 \\
& \mathrm{D} 5 y(t)=[\mathrm{D} 1 y(t)+2 \mathrm{D} 2 y(t)] / 3
\end{aligned}
$$

As described by these equations, the leftmost dot (D1) in each display oscillated vertically. The other dots had two elements of motion: one was the same vertical oscillation as D1, and the other was a pendular oscillation around $\mathrm{Dl}$; that is, the motion of a moving endpoint of a pendulum. The frequency of motion was kept con-

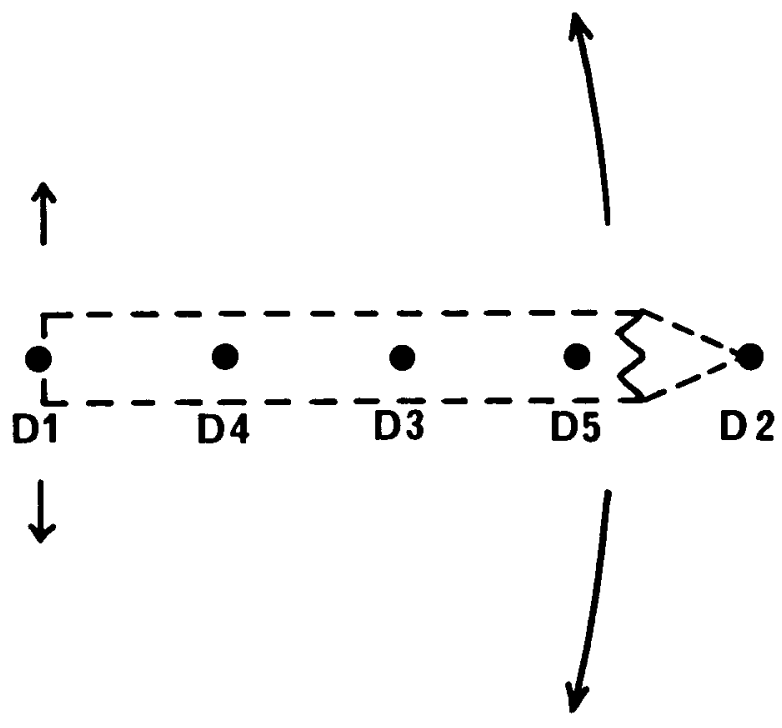

Figure 1. A sample configuration of stimulus dots moving in the frontal parallel plane. The left dot (D1) moved in vertical simple oscillation. The other dots (D2, D3, D4, and D5) moved simultaneously in both vertical simple oscillation and pendular oscillation around the left dot (D1). The broken lines indicate a simulated rigid pencil that was not presented in the actual displays. Dots D1 and D2 corresponded to its endpoints. stant at $1.5 \mathrm{~Hz}$ (angular frequency, $w$ in the equations, was $3 \pi$ ). The amplitude of the pendular oscillation ( $s 2$ in Equations $1-3$ and 1-4) was held constant at $30^{\circ}$.

The 25 displays resulted from the following conditions.

1. Number of dots: We investigated the effect of number of dots on the perceived structure of an interpolated rod among dots. Two (D1 and D2), three (D1, D2, and D3), or four (D1, D2, D4, and D5) dots were presented on the screen. The amplitude of vertical oscillation ( $a$ in the equations) was set at $1.2^{\circ}$ in visual angle, and the distance (i.e., the length of a simulated rigid rod) between D1 and D2 ( $d$ in the equations) was set at $6^{\circ}$ under this condition. Hereafter, these stimuli are referred to as the standard stimuli.

2. Reduced scale of rod: We also investigated a reduced scale effect of the simulated rod. Only two dots (D1 and D2) were presented under this condition. The reduced scale was either $2 / 3$ or 1/3 of that of the standard stimuli. As a result, the amplitude of vertical oscillation was $48^{\prime}$ or $24^{\prime}$ in visual angle, and the distance between the two dots was $4^{\circ}$ or $2^{\circ}$.

These conditions were combined with a variation in the phase difference ( $s 1$ in Equation 1-2) of the vertical oscillation versus that of the pendular oscillation. This phase difference was set at $0^{\circ}, 45^{\circ}, 90^{\circ}, 135^{\circ}$, or $180^{\circ}$. The phase difference resulted in a similar phase difference between the motion of the left dot (D1) and that of the right dot (D2).

Experiment 1B: Velocity Factor. A set of 50 displays was presented in random order. Each display was presented three times. The standard stimuli (i.e., D1 and D2, the distance between which was $6^{\circ}$ ) from Experiment $1 \mathrm{~A}$ were used. The 50 displays resulted from the orthogonal variation of two factors: the frequency ( $w$, or angular frequency, in the above equations), which was varied from 0.2 to $2.0 \mathrm{~Hz}$ by $0.2-\mathrm{Hz}$ steps, and the phase difference ( $s 1$ in the equations), which was also varied among $0^{\circ}, 45^{\circ}, 90^{\circ}, 135^{\circ}$, and $180^{\circ}$.

In both experiments, the size of the moving dots was $1^{\prime}$ in visual angle, and their luminance was $7.8 \mathrm{~cd} / \mathrm{m}^{2}$. No fixation point was placed, and the subjects could move their eyes freely.

\section{Procedure}

The same subjects participated in both experiments: the experiment on spatial factor first, followed by the experiment on velocity factor. The task that the subjects were required to perform was the same in both experiments. The subjects were asked to judge whether the dots appeared to move as if they constituted one moving rod. Furthermore, if the subjects perceived the moving dots as constituting a rod, they were required to rate the elasticity of the structure. The subjects used a scale in which 1 indicated complete rigidity and 99 indicated maximum elasticity. It was not difficult for the subjects to rate the elasticity after 25 practice trials in each session.

\section{Results}

\section{Experiment 1A: Spatial Factor}

Out of 1,125 responses, only 8 indicated that the moving dots appeared to be independent. Thus, the subjects perceived the moving dots as constituting a moving rod on almost every trial.

It is striking that the dots were perceived as an elastic structure, and not as a rigid one, on almost every trial. Typical examples of perceived elastic structures are shown in Figure 2. The solid lines indicate this subjective elastic structure, and the broken lines indicate the simulated wiggling rigid rod. Figures $3 a$ and $3 b$ show the mean elasticity rating of the subjective rod as a function of the phase difference between the vertical simple oscillation 

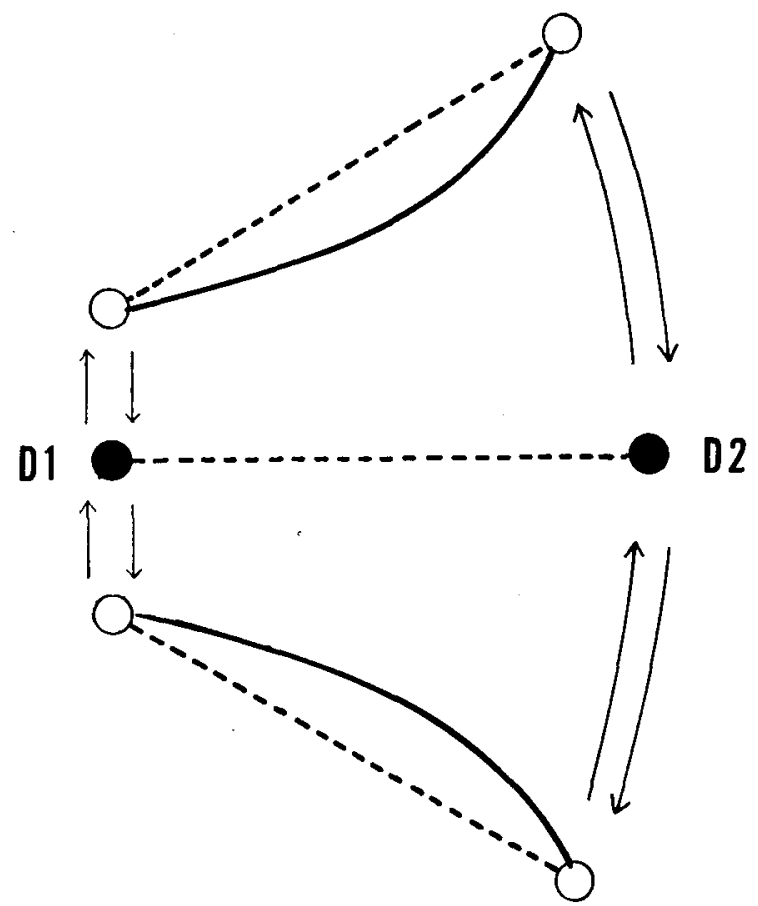

Figure 2. A typical example of the perceived elastic structure induced by moving dots under the condition of two-dot motion. The phase difference between the vertical oscillation and the pendular oscillation is $0^{\circ}$. Solid lines indicate the perceived elastic rod, and broken lines indicate the simulated rigid rod.

and the pendular oscillation. Figure 3a shows the effect of the number of moving dots, and Figure $3 \mathrm{~b}$ shows the effect of reduced scale. The findings presented in these figures suggest that the subjects perceived the elastic rod regardless of both the number of dots and the reduced scale of the stimulus. However, the degree of perceived elasticity tended to decline with the number of dots. The

a

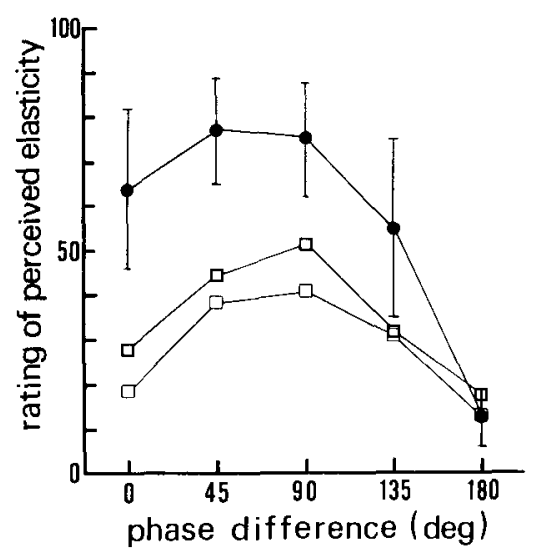

subjects reported that the subjective rod was perceived to bend, particularly at a part near D1 of the rod under the three- and four-dot conditions. These findings reveal the strong effect of phase difference on perceived elasticity. Analyses of variance (ANOVAs) on elasticity ratings were performed separately to determine the effects of the number of dots and of reduced scale. The ANOVAs showed a significant effect of number of dots $[F(2,210)$ $=59.6, p<.001]$, a significant effect of phase difference $[F(4,210)=37.0, p<.001]$, and a significant interaction between number of dots and phase difference $[F(8,210)=5.2, p<.01]$. This significant interaction may reflect the observation that a rigid rod was often perceived, regardless of the number of dots, when the phase difference was $180^{\circ}$. In addition, the reduced scale had a significant effect on the perceived elasticity of the object $[F(2,210)=4.33, p<.05]$, and the effect of the phase difference was significant $[F(4,210)=86.3$, $p<.001]$. Furthermore, in an examination of the relationship between the influence of the number of dots and that of the reduced scale, a comparison of the data illustrated in Figures $3 a$ and $3 b$ revealed a significant difference between the condition in which the scale was most reduced and the condition of four-dot motion $[F(1,140)$ $=52.6, p<.001]$. This is important because the distance between dots was the same in both conditions.

\section{Experiment 1B: Velocity Factor}

Out of 2,250 responses, only 15 indicated that the motion of the dots appeared to be independent. Figure 4 shows the mean elasticity ratings as a function of oscillation frequency. The subjects perceived vivid elastic structures in the dot motion, regardless of the frequency of oscillation, except for the condition in which the phase difference was $180^{\circ}$. An ANOVA showed a significant effect of velocity variation on perceived elasticity

b

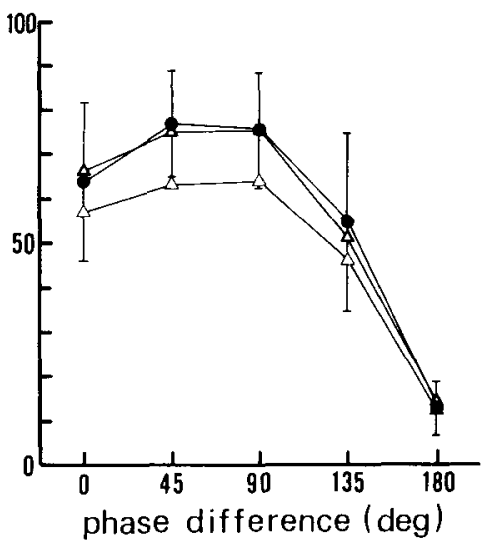

Figure 3. The mean elasticity rating of the interpolated rod between the dots as a function of the phase difference between the vertical oscillation and the pendular oscillation. (a) The number of dots was varied: closed circles indicate the standard two-dot stimuli in motion; heavy-outline squares, three dots in motion; thin-outline squares, four dots in motion. (b) The scale was varied: closed circles indicate the standard stimuli in motion; heavyoutline triangles, $2 / 3$ of the reduced scale; thin-outline triangles, $1 / 3$ of the reduced scale. Error bars indicate the standard deviation. 


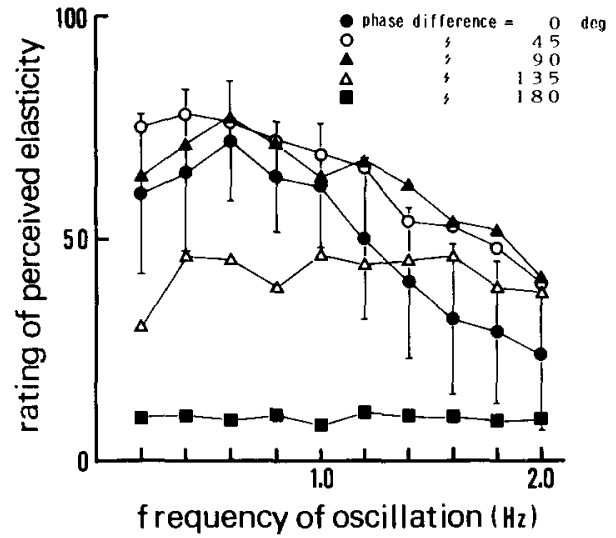

Figure 4. The mean elasticity rating of the interpolated rod between the two moving dots as a function of the oscillation frequency of the dots. Closed circles indicate the condition of $0^{\circ}$ phase difference; open circles, $4^{\circ}$; closed triangles, $90^{\circ}$; open triangles, $135^{\circ}$; closed squares, $180^{\circ}$.

$[F(9,700)=18.2, p<.001]$ and phase difference $[F(4,700)=301.4, p<.001]$, and a significant interaction between these effects $[F(36,700)=4.1, p<.01]$. As shown in Figure 4, the degree of perceived elasticity tended to decline as the velocity was increased. This decline was greatest in the condition in which the phase difference was $0^{\circ}$.

\section{Discussion}

The results in this experiment can be summarized as follows:

1. Two moving dots were sufficient to produce an apparent moving rod whose endpoints corresponded to these dots. This was shown by the finding that almost all responses under every condition indicated that the moving dots appeared to constitute a rod.

2 . The simulated rigid rod was not perceived as being rigid under many conditions; instead, an elastic rod was perceived. The apparent structure of the perceived moving rod was similar to that of a thin elastic rod vibrating transversely, as shown in Figure 2.

3. The phase difference between the oscillatory motions had strong effects on the perceived elasticity of the interpolated rod. It should be noted, however, that a high degree of elasticity was observed even when the phase difference was $0^{\circ}$. On the other hand, a vivid elastic structure was almost never observed under the condition in which the phase difference was $180^{\circ}$. This might have been caused partly by the misperception of the location of the center of the pendular oscillation: the subjects reported that this center was perceived at a point on the simulated rod other than the position of the actual center of this oscillation (i.e., the position of D1). The motion of two dots, when oscillating completely out of phase, looked like a seesaw or a lever. The human visual system can, in general, correctly detect 3-D parameters (e.g., time to collision [Lee, 1976, 1980] and edge types [Clock- sin, 1980]) of moving elements that constitute 3-D objects. However, in this experiment, the subjects misperceived the center of rotation, even though they recognized that the left dot moved in a vertical simple oscillation and not in a pendular oscillation. Thus, an interpolation might have been performed among three colinear points (i.e., two actual dots and the perceived center of pendular oscillation) that resulted in the perception of the rigid rod; however, in most conditions, this did not happen.

4. The subjective rod was constructed more elastically from the motion of two dots than from the motion of three or four dots. This can be confirmed by the findings of Green (1961) that the perceived coherent structure of an object from the motion of its elements is affected by the number of elements. In this regard, the reduced distance between dots had no crucial role. The motion of two dots with the most reduced scale was perceived as a very elastic rod. On the other hand, the four-dot motion with the same distance between dots as that in the condition of the most reduced scale was perceived as a much less elastic rod. Thus, it cannot be contended that the reduced distance caused the coherent structure. As a result, it can be concluded that the mechanism for the recovery of the rigid structure is more effective in three- or four-dot motion than in two-dot motion.

5 . The reduced scale of the simulated rod had a small effect on the perceived structure. When the scale was reduced to $1 / 3$, the perceived elasticity of the interpolated rod decreased. However, a vivid elastic structure was still reported, even under this most reduced scale condition, in which the amplitude of the vertical oscillation was 24 ' and the distance between two dots was $2^{\circ}$. In this condition, the subject could observe the display within the fovea, which suggests that the eccentricity on the retina had little effect on perceived elastic structure.

6 . The velocity of dot motion had some effect on perceived elasticity. The variable used in the experiment was the oscillation frequency of the dots; the distance between dots was kept constant. Thus, the variation of the frequency corresponded to that of the velocity. The effect of velocity in this experiment can be described as follows: The degree of perceived elasticity of the interpolated structure decreased as the velocity was increased within the range of the velocity variation used in this experiment, except in the condition in which the phase difference was $180^{\circ}$. The mechanism of the effect of velocity on perceived elasticity is still uncertain, but it is important that the elastic structure was perceived from motion with very low velocity.

With these results, we must consider why and how the elastic structure can be perceived. A certain mechanism must operate on information in the retinal image.

In the case of the normal rubber pencil illusion, as Pomerantz (1983) hypothesized, visual persistence occurring early in the visual system could induce the perception of elastic motion of the pencil. On the other hand, the perceived elastic structure in this study was not related to visual persistence. This is because no curved structure 
persisted on the retinal image in this experiment. It is possible, however, that visual persistence in higher processes - the imagery processes-is involved in the perception of this elastic structure. In this case, two successive processes may operate. First, a rigid rod is interpolated between the two moving dots at each moment in time. These rigid rods then persist in the memory representation, resulting in the construction of curved structures, as in the rubber pencil illusion. However, these processes of image persistence are doubtful. The results of Experiment 1 showed that a vivid bending structure was observed when the phase difference was $0^{\circ}$, a condition in which persistence could not produce a curved image in higher visual processes, such as retinal processes.

It should be examined here whether the visual system extracted the external information accurately in this experiment. Although the motion of a rigid rod was simulated, the observers might have registered the motion of a certain elastic rod because of inaccurate extraction. Concerning this, there are two possible kinds of information for the recovering of the elastic structure from the motion of two dots.

One possibility is the phase difference between dot motions. There is a physical axiom about the motion of an elastic body: When an elastic object oscillates, some phase differences occur among the oscillations of its elements. Although the reverse of this axiom is not always true, our visual system may tend to respond as if it were; when it registers a phase difference, we may perceive an elastic structure. This is so in particular when the information about the motion of the object's elements is limited as in this experiment. Certainly, the results of Experiment 1 showed that a vivid elastic structure was observed under the condition of no phase difference between the motion of the dots. It is possible, however, that the inaccurate extraction of information about dot motion causes the visual system to register a phase difference, even when there is no stimulus phase difference. This inaccurate extraction of information may be due to the factor of spatial distance (i.e., eccentricity on the retina) or the velocity of moving dots. That is, when dots move with a large separation in space or at a high velocity, the extraction of their motion cannot be done in parallel, but must be done serially, which may result in some phase differences between the motions of the dots. Nevertheless, the results of Experiment 1 are counterevidence to this possibility: when two dots moved within $2^{\circ}$ in visual angle, or at the minimum velocity $(0.2 \mathrm{~Hz})$, higher ratings for the elasticity of interpolated structure were obtained.

The other possibility may be the distance between moving dots. When a rigid rod moves in a frontal parallel plane, the distance between its elements in the retinal image is kept constant. On the other hand, for the elastic rod in motion, this constancy of retinal distance does not hold. Considering this point, it is possible that the subjects perceived the distance between moving dots to vary in Experiment 1, even though it was physically constant. This perception of changing distance may have, in turn, induced the perception of the elastic structure. A widely known and relevant visual phenomenon is the Ansbacher effect (Ansbacher, 1944): When a dot rotates around a point, its trajectory is perceived to be smaller than its physical trajectory. Thus, the subjects might have perceived a shrinking of the distance between the dots, and they might have recovered the elastic structure from this shrinking motion.

Experiment 2 was conducted to examine this possibility. The distance between two moving dots was varied in this experiment. When a rod is physically bent, the distance between its two endpoints decreases. Yet, in this experiment, the projected distance between two dots moving in depth was increased, although the distance in depth between them was kept constant (Figures $5 a$ and 5b). If a bending structure is perceived even under this condition, the shrinking of the perceived distance could not have produced the perceived bending structure in Experiment 1.

\section{EXPERIMENT 2}

\section{Method}

\section{Subjects}

Ten students (4 graduates and 6 undergraduates) served in this experiment. All subjects had participated in Experiment 1 or in the pilot study that preceded Experiment 1. They all had normal or corrected-to-normal visual acuity.

\section{Apparatus}

The apparatus used in this experiment was the same as that used in Experiment 1.

\section{Stimuli}

A set of 36 displays was presented in random order. Each display was presented three times. The motion of the dots presented on the CRT can be described by the following equations:

$$
\begin{aligned}
& \mathrm{D} 1 x(t)=\text { constant } \\
& \mathrm{D} 1 y(t)=a \sin (w t+s 1) \text { or constant } \\
& \mathrm{D} 2 x(t)=\mathrm{D} 1 x(t)+d \cos (s 3) \cos [s 2 \sin (w t)] \\
& \mathrm{D} 2 y(t)=\mathrm{D} 1 y(t)+d \sin [s 2 \sin (w t)]
\end{aligned}
$$

The 36 displays resulted from the combination of the following conditions:

1. Rotation angle in depth: The moving dots (D1 and D2) presented on the CRT (P2 in Figure 5a) were the orthogonal projected images of the dots moving in depth, as shown in Figure 5a. The plane (P1 in Figure 5a) on which the dots moved had some rotation angle in depth ( $s 3$ in Figure $5 \mathrm{a}$ and in the above equations) relative to the frontal parallel plane. This angle was varied from $0^{\circ}$ to $80^{\circ}$ by $10^{\circ}$ steps.

2. Phase difference: The phase difference ( $s 1$ in the equations) between two elements of the motion of the right dot (D2) was produced in the same way as in Experiment 1. The phase differences used in this experiment were $0^{\circ}, 90^{\circ}$, and $180^{\circ}$. The condition that D1 was stationary was added in this experiment. There were no phase differences in this condition. Thus, in this experiment, there were four conditions, one with no motion of D1 and three with motion of both dots in different phase relations.

Under all conditions, the frequency of the motion was kept constant at $1 \mathrm{~Hz}$ (angular frequency, $w$ in the equations, was $2 \pi$ ); the 


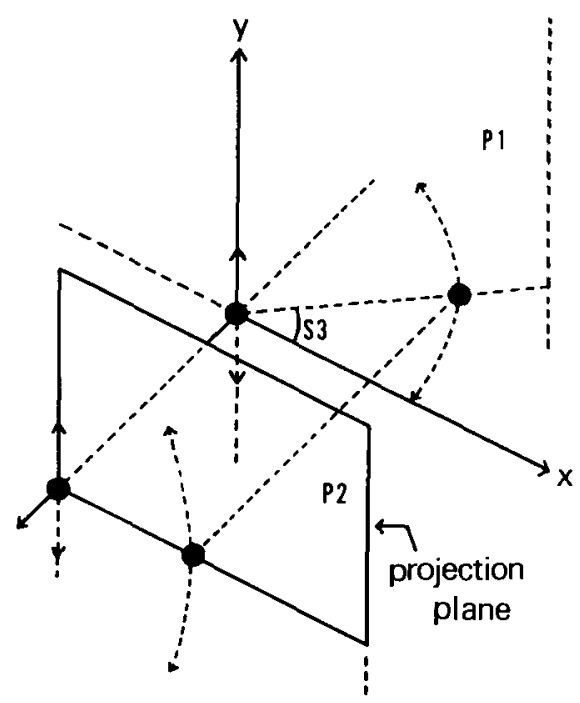

b

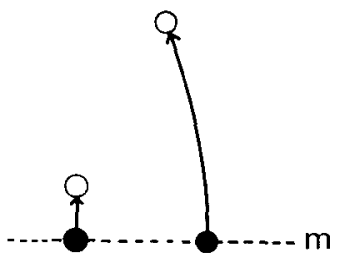

Figure 5. (a) The configuration of the stimuli in Experiment 2. The projection plane (P2) was parallel vertically to the tilted plane (P1) in which the dots moved. Plane P1 was rotated about a vertical axis by an angle s3 relative to the frontal plane (P2). (b) The trajectory of a quarter cycle of the moving dots in plane P2. Dotted lines indicate the axis of symmetry (the horizontal axis in this experiment).

amplitude of the vertical oscillation ( $a$ in the equations) was $1.2^{\circ}$ in visual angle; the distance between dots ( $d$ in the equations) was $6^{\circ}$ in visual angle; the amplitude of the pendular oscillations ( $s 2$ in the equations) was $30^{\circ}$; and the luminance of the stimuli was $7.8 \mathrm{~cd} / \mathrm{m}^{2}$.

\section{Procedure}

The subjects were asked to allocate the appearance of the motion of the two dots into the following nine categories:

0 . The two dots appear to move separately, and do not appear to constitute any object.

1. The two dots appear to constitute a rigid rod moving in the frontal parallel plane.

2. The two dots appear to constitute a stretching rod moving in the frontal parallel plane.

3. The two dots appear to constitute a bending rod moving in the frontal parallel plane.

4. The two dots appear to constitute a stretching and bending rod moving in the frontal parallel plane.

11. The two dots appear to constitute a rigid rod moving in depth.

12. The two dots appear to constitute a stretching rod moving in depth.

13. The two dots appear to constitute a bending rod moving in depth.

14. The two dots appear to constitute a stretching and bending rod moving in depth.

The subjects performed nine practice trials in which the display, which included one of the nine rotation angles, was presented.

\section{Results and Discussion}

The dot motion used in this experiment was the projected motion of elements constituting a moving rigid rod in depth. Thus, it was predicted that the observers would perceive a rigid rod moving in depth if they adequately extracted the depth information from the motion of the dots in the frontal parallel plane. If the observers extracted the depth information inadequately, they were expected to perceive a stretching rod. This is because the distance between moving dots in the plane was varied in this experiment, except in the condition of $0^{\circ}$ depth rotation angle of plane P1.

Figure 6a shows the rates of depth perception obtained in each phase difference condition and in the condition in which the dot was stationary. These values are given as a function of the cosine of the depth rotation angle of plane $P 1$, and they are the proportion of the frequency of Categories $11,12,13$, and 14 to the total frequency. As shown in this figure, the observers tended to extract the increasing depth information from the motion of the dots in a frontal plane more frequently with the increase of the depth rotation angle of plane P1. This depth information was thought to be the changing distance between dots as a function of the cosine of the depth rotation angle of plane $P 1$.

Figure $6 \mathrm{~b}$ reveals that the observers perceived the bending rod interpolated between dots as they did in Experiment 1 , when they extracted the depth information from the motion of the dots. In this figure, the rates of the perceived bending motion correspond to the proportion of the frequency of Categories $3,4,13$, and 14 to the total frequency. As shown in Figure $6 \mathrm{~b}$, it is clear that the rate of the perceived bending motion decreased as the depth rotation angle of plane P1 increased. Yet the rate was still high, and particularly so under the condition in which the phase difference was $90^{\circ}$.

Two important results should be discussed here.

First, a vivid bending structure was perceived even when the distance between the two dots increased as they moved away from the horizontal plane. In this experiment, the distance between dots increased after the right dot 


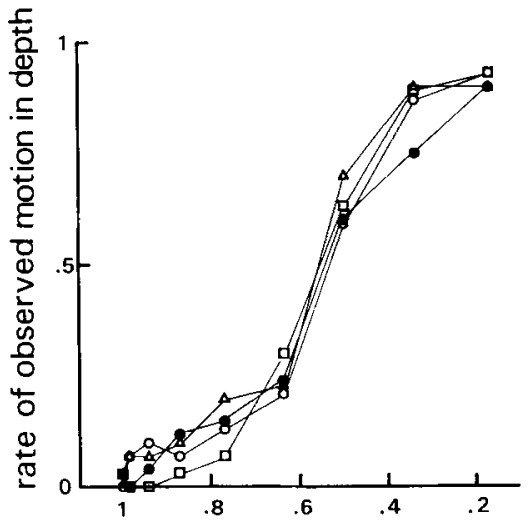

cosine of rotation angle in depth b

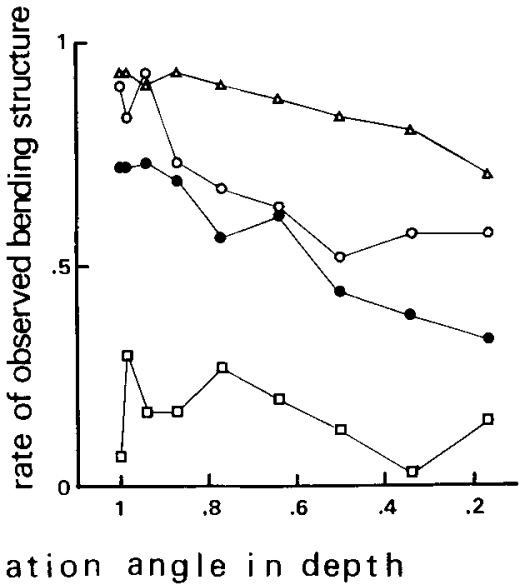

Figure 6. (a) The rate of observed motion in depth as a function of the cosine of the depth rotation angle of plane $\mathrm{P1}$. The rates were those of the frequency of Categories 11 , 12, 13, and 14 to the total frequency. (b) The rate of the perceived bending structure as a function of the cosine of the depth rotation angle of plane $P 1$. The rates were those of the frequency of Categories $3,4,13$, and 14 to the total frequency. In both figures, open circles indicate the condition of $0^{\circ}$ phase difference; open triangles, $90^{\circ}$; open squares, $180^{\circ}$. Closed circles indicate the condition in which the left dot was held stationary.

passed across the axis ( $m$ in Figure $5 b$; i.e., the horizontal axis) of symmetry of the trajectory. The observers tended to perceive a depth or a stretching motion in response to the increment of the distance, and to simultaneously perceive the bending structure. This phenomenon, however, is inconsistent with the physical axiom about the elastic object. When an elastic object oscillates and bends (not stretches), the distance between its elements decreases. It would not be inconsistent with the physical constraint if the observers perceived the bending structure in response to a decrement of the distance between dots. From this result, therefore, we can conclude that the distance between dots had no crucial role in inducing the perception of a bending structure in the experiments reported here.

Second, even when the left dot was fixed and stationary, a vivid bending structure was perceived. From this result, we can conclude, as described before, that a phase difference between motions is not the crucial factor inducing the perception of a bending structure between dots. Under this condition, some subjects reported an induced motion of the left fixed dot produced by the right moving dot. In the case of the induced motion, however, the phase difference between the induced motion and the inducing motion is $180^{\circ}$. As shown in the results of the present experiments, no bending structure was perceived under the condition in which the phase difference was $180^{\circ}$. Therefore, the phase difference caused by the induced motion had no positive role in the perceived bending structure.

\section{GENERAL DISCUSSION}

The purpose of the present study was to examine the plausibility of a suggestion made by Hoffman and Flinch- baugh (1982). Their suggestion was that the human visual system has the ability to recover a rigid rod from the motion of the rod's two end elements. The present experiments investigated a widely known phenomenon, the rubber pencil illusion (Pomerantz, 1983). That is, when a rigid rod is held loosely at one end and wiggled it appears to have a nonrigid structure. The purpose of these experiments was to determine how a rigid rod delineated only by its endpoints is perceived when wiggled. When two dots constituting one rigid rod oscillated as the two endpoints of a pencil in the manner that produces the rubber pencil illusion, the problem was whether the perceived structure is a rigid rod, as suggested by Hoffman and Flinchbaugh (1982), or a nonrigid one, as in the rubber pencil illusion. The results of Experiment 1 show that a nonrigid bending structure can be observed from the motion of two dots. Therefore, the suggestion made by Hoffman and Flinchbaugh was disconfirmed.

A possible explanation for this perceived bending structure was considered. The display that included two moving dots in these experiments might have been registered inaccurately so as to correspond the same information as that produced by an elastic rod. If this explanation is feasible, it is plausible that the visual system constructs the bending structure using this information. However, this explanation was disconfirmed by the results of the experiments reported here. Both the distance and the phase difference information, which are associated with a physically bending structure, were not crucial in these experiments. This was shown by the result that a vivid bending structure was observed even when the distance between two dots increased as they moved away from the horizontal plane. Other results showed that a vivid structure was observed even under the condition in which the phase difference between the motions of the dots was $0^{\circ}$, as well 
as under the condition in which one dot was stationary and the other moved.

These results suggest that other mechanisms must be responsible for inducing the perception of a bending structure from two moving dots that simulate the motion of the endpoints of a rigid rod. When elucidating the underlying mechanisms, we should consider the results of the present study: that these mechanisms are under some influence of both the number of moving dots (Experiment 1) and the phase difference between the motions of the dots (Experiments 1 and 2). In particular, the latter has an important effect on the elasticity of the perceived structure, although bending can be perceived in the absence of a phase difference.

\section{REFERENCES}

Ansbacher, H. L. (1944). Distortion in the perception of real movement. Journal of Experimental Psychology, 34, 1-23.

Clocksin, W. F. (1980). Perception of surface slant and edge labels from optical flow: A computational approach. Perception, 9, 253-269.

GREEN, B. F. (1961). Figure coherence in the kinetic depth effect. Journal of Experimental Psychology, 62, 272-282.
Hoffman, D. D., \& Flinchbaugh, B. E. (1982). The interpretation of biological motion. Biological Cybernetics, 42, 795-204.

Johansson, G. (1973). Visual perception of biological motion and a model for its analysis. Perception \& Psychophysics, 14, 201-211.

JoHansson, G. (1975). Visual motion perception. Scientific American, 232, 76-88.

LEE, D. N. (1976). A theory of visual control of braking based on information about time to collision. Perception, 5, 437-459.

LEE, D. N. (1980). The optic flow field: The foundation of vision. Philosophical Transactions of the Royal Society of London, Series B, 290, 169-179.

Pomerantz, J. R. (1983). The rubber pencil illusion. Perception \& Psychophysics, 33, 365-368.

TSAI, R. Y., \& HUANG, T. S. (1981). Uniqueness and estimation of three-dimensional motion parameters of rigid objects with curved surfaces (Coordinated Science Laboratory Report R-921). Champaign, IL: University of Illinois.

Ullman, S. (1979). The interpretation of visual motion. Cambridge, MA: MIT Press.

UllmaN, S. (1983). Recent computational studies in the interpretation of structure from motion. In J. Beck, B. Hope, \& A. Rosenfeld (Eds.), Human and machine vision. New York: Academic Press.

Wallach, H., \& O'ConNell, D. N. (1953). The kinetic depth effect. Journal of Experimental Psychology, 45, 205-217.

(Manuscript received October 11, 1986; revision accepted for publication October 17, 1987.) 